\title{
Analysis and Evaluation of a Heat Integrated Horizontal Distillation System
}

\author{
Papadakis, Emmanouil; Mansouri, Seyed Soheil; Huusom, Jakob K.; Abildskov, Jens
}

Published in:

Chemical engineering transactions

Link to article, DOI:

10.3303/CET1869108

Publication date:

2018

Document Version

Publisher's PDF, also known as Version of record

Link back to DTU Orbit

Citation (APA):

Papadakis, E., Mansouri, S. S., Huusom, J. K., \& Abildskov, J. (2018). Analysis and Evaluation of a Heat Integrated Horizontal Distillation System. Chemical engineering transactions, 69, 643-648.

https://doi.org/10.3303/CET1869108

\section{General rights}

Copyright and moral rights for the publications made accessible in the public portal are retained by the authors and/or other copyright owners and it is a condition of accessing publications that users recognise and abide by the legal requirements associated with these rights.

- Users may download and print one copy of any publication from the public portal for the purpose of private study or research.

- You may not further distribute the material or use it for any profit-making activity or commercial gain

- You may freely distribute the URL identifying the publication in the public portal 


\title{
Analysis and Evaluation of a Heat Integrated Horizontal Distillation System
}

\author{
Emmanouil Papadakis, Seyed Soheil Mansouri, Jakob K. Huusom, Jens \\ Abildskov* \\ PROSYS, Department of Chemical and Biochemical Engineering, Technical University of Denmark, Søltofts Plads, Building \\ 229, DK-2800 Kgs. Lyngby, Denmark \\ ja@kt.dtu.dk
}

\begin{abstract}
We present a model-based approach to analyze a heat integrated horizontal distillation system for separating mixture of light alcohols from second-generation biomass. The objective is to highlight the application of an engineering tool developed based on process system engineering methods. The tool is used to analyze and evaluate the proposed separation system and to provide guidelines with respect to specific design and process variables. The model is used to perform sensitivity analysis studies on important variables such as mass and heat transfer coefficients, interfacial area and to perform simulation studies to investigate the sensitivity system performance to heat transfer area, product purity and recovery. The objective of the sensitivity analysis of the transport coefficients is to show the effect of possible deviations on the performance of the process and to provide detailed knowledge. The analysis has shown that changes in transport coefficients do not affect the performance of the system unless there are significant deviations $(>80 \%)$. Simulation studies in heat transfer area show that increased heat transfer area leads to process improvements in terms of energy consumption.
\end{abstract}

\section{Introduction}

Production of chemicals from second-generation (2G) feedstock requires gathering adequate feedstock for one central plant (Maity et al. 2015), which can be challenging. This makes small-scale factories based on local flexible production an attractive alternative. Here, a small-scale modular separation system tailor-made for mobile refineries is explored, to enable fast and cost-effective manufacturing of customized products at various locations. Therefore, a container format that is easily configured for different products and processes is considered here. The container format allows on-site transportation to provide manufacturing anywhere, enabling the benefits of localized service delivery without duplication of equipment at multiple locations. We focus on the development of separation technology for the separation of mixtures containing light alcohols from small-scale production facilities of alcohols from 2G biomass (such as straw and wood chips). The paper deals with the application of a steady-state model that serves as a tool, which in absence of experimental data, describes adequately the separation system and perform analysis and evaluation in terms of process performance (Papadakis et al. 2018). The proposed horizontal separation system aims at reduction of the costs (such as capital cost and operating cost) associated with conventional distillation systems and novel intensified and highly integrated designs such as reactive distillation, diabatic distillation, internally heat integrated distillation column (HIDiC) and the divided wall column (Kiss et al. 2013). The feasibility of other horizontal distillation systems, in separating binary liquid mixtures, has been investigated by experimental and numerical simulation studies (Seok et al. 1985, Ramirez-Gonzalez et al. 1992). Recently, Kim et al. (2013) and Jang et al. (2015) have shown, experimentally and numerically, the potential energy savings using a diabatic rectangular horizontal column in liquid binary mixtures. The focus here is the development of a separation system that is horizontal for the separation of light alcohols from small-scale production facilities. The proposed design consists of two sections, i.e. a stripper and a rectifier. The system has no trays. Therefore, the model is rate-based. It operates in continuous mode, it can handle viscous liquids (or liquids containing solids), high-value temperature sensitive components and offers opportunities for heat integration. A steady 
state model based on the two-film theory of mass transfer has been previously developed and has been used to demonstrate that the model qualitatively describes the system (Papadakis et al. 2018). This steady state model has been developed to qualitatively represent the real system and capture the gross features of it. The model has been applied in conceptual analysis studies, and it has shown the potential energy savings compared to conventional separation technologies and that the proposed distillation technology is beneficial for mixtures containing low amounts of alcohols (for example, 10 wt.\%) (Papadakis et al 2018). Here, the system and the developed model is briefly described, then the application of the model is highlighted in sensitivity analysis studies of the transport coefficients and in simulation scenarios. Finally, conclusions are made, highlighting the need of an economic model.

\section{System description}

The diabatic distillation system is illustrated in Figure 1. It consists of two, co-axially arranged circular horizontal tubes. The inner tube operates as a stripper and is placed inside the outer one that operates as a rectifier. The feed consists of light alcohols, water and temperature sensitive compounds and enters the stripping section at reduced pressure preventing decomposition of the temperature sensitive compounds. The heavy product of the stripping section consists of water and valuable compounds and the light product consists of more light alcohols. The stripper light product is compressed and charged into the rectifying section, where the alcohols are concentrated and collected as light product, whereas the bottom product is returned to the stripper after expansion. Heat integration is thereby made possible between the rectifying and the stripping chambers.

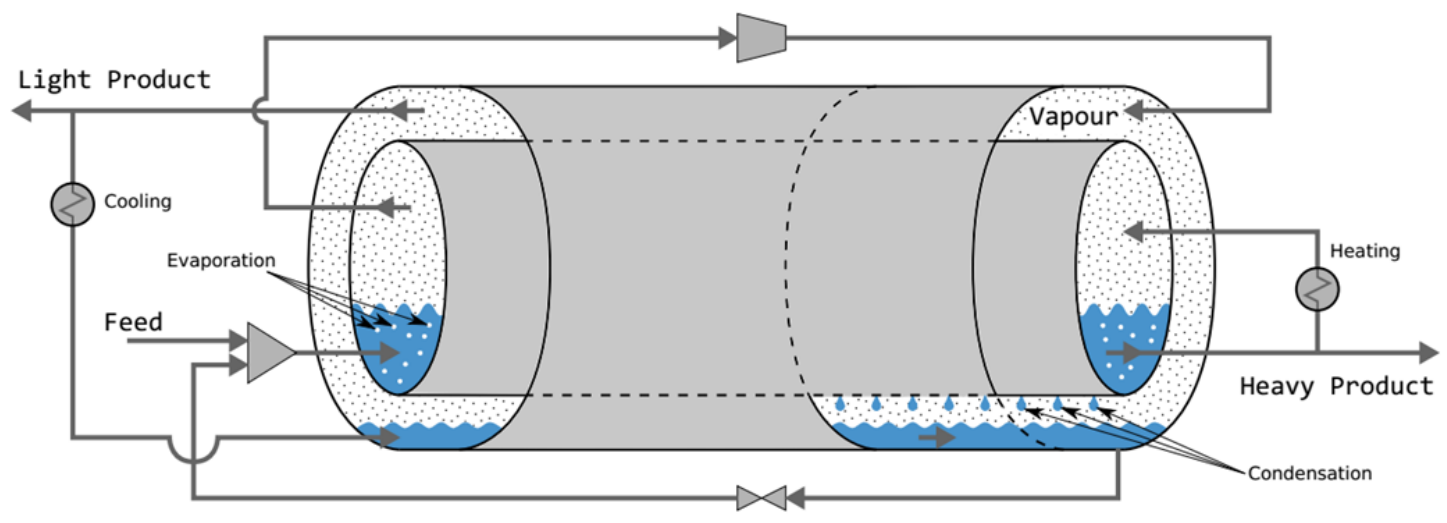

Figure 1: The heat integrated horizontal distillation system. The stripper is represented by the inner chamber and the rectifier is represented by the outer chamber. In the stripper and rectifier, evaporation and condensation takes place, respectively. Dimensions: tube length $=2 \mathrm{~m}$, stripper diameter $=1.8 \mathrm{~m}$ and rectifier diameter $=2 \mathrm{~m}$.

\section{Model development}

Papadakis et al. (2018) have described the development of the steady state model. The objective of the model is to describe sufficiently the fundamental phenomena taking place in the horizontal distillation system and it needs to predict qualitatively, for both chambers, the internal flow rate profiles, the temperature and the composition profiles in the vapor and liquid phases. The steady state model is to be used as a tool, which in the absence of experimental data and low technology awareness, may assist in conceptual analysis and evaluating preliminary process designs, and for comparative and optimization studies. The model developed considers the following phenomena: Rate-based separation, counter-current flow of vapor and liquid phases in both chambers, heat transfer between rectifier and stripper and compression of the stripper's exiting vapor and pressure reduction of the rectifiers (outlet) liquid. For the model development the following assumptions have been made: Vapor-liquid equilibrium is assumed only at the interface, the pressure of the rectifier and stripper is perfectly controlled, the liquid remains on the surfaces, the reflux ratio and the boil-up ratio are perfectly controlled. Heat transfer coefficient is calculated using the Chilton/Colburn correlation (Taylor, R., Krishna, 1993). Mass transfer coefficients and interfacial area are calculated using the Bravo et al. (1985) correlation. Finally, the Wilson activity coefficient model has been applied as the thermodynamic model. The model has been constructed using segments and represented by MERSHQ (Material-Energy-RateSummation-Hydraulic-Equilibrium) equations, a reboiler and condenser (as equilibrium stages), a compressor and a valve and finally, a heat transfer model between the stripping and rectifying section (Eq. 1). 


\subsection{Heat Integration}

Heat integration takes places between the rectifier and the stripper. The rectifier operates at higher pressure, to ensure sufficient temperature difference. The heat transferred from the rectifier to the stripper per segment $j$ is:

$Q_{j}=n \cdot U \cdot A_{j} \cdot \Delta T_{j}$

Here $U$ is the overall heat transfer coefficient in $\frac{k W}{m^{2} K} . A_{j}$ is the heat transfer area in $\mathrm{m}^{2} . \Delta T_{j}$ is the temperature difference between the rectifier and the stripper, $\Delta T_{j}=T_{R, j}-T_{S, j}$ (in $\mathrm{K}$ ) and $n$ is a factor that takes positive non-zero integer values $(1,2,3, \ldots)$. It is used to multiply the heat transfer area $U A_{j}$ in order to manipulate its value. For the overall heat transfer coefficient, a value of $1 \frac{\mathrm{kW}}{\mathrm{m}^{2} \mathrm{~K}}$ has been chosen. The heat transfer area has been calculated considering that the minimum transfer area $\left(A_{j}\right)$ per segment $j$ is the surface of a stripping segment $j$.

\section{Case study: Ethanol-water mixture}

The mixture of ethanol-water has been selected to highlight the application of the developed steady-state model for the horizontal distillation system. That mixture is common after fermentation of $2 \mathrm{G}$ biomass together with temperature sensitive compounds (such as enzyme), although such compounds are not considered in this simplified treatment. Table 1 lists the values of the variables specified to solve the model.

Table 1: Process variables used for the simulation of the heat integrated horizontal system.

\begin{tabular}{llll}
\hline Process Variables & Values & Process Variables & Values \\
\hline Inlet flow rate & $0.1 \mathrm{kmol} / \mathrm{sec}$ & Rectifier pressure & $0.3 \mathrm{~atm}$ \\
Inlet Temperature & $40{ }^{\circ} \mathrm{C}$ & Reflux ratio & 2.5 \\
Inlet Pressure & $0.1 \mathrm{~atm}$ & Boil-up ratio & 0.15 \\
Ethanol composition & $10 \mathrm{wt} .-\%$ & Phase contact area, S & $50 \mathrm{~m}^{2} / \mathrm{m}^{3}$ \\
Stripper pressure & $0.1 \mathrm{~atm}$ & Phase contact area, R & $50 \mathrm{~m}^{2} / \mathrm{m}^{3}$ \\
\hline
\end{tabular}

\section{Sensitivity analysis-Transport coefficients}

The objective here is to show the effect of the transport coefficients that dictate the mass transfer between the rectifying and stripping section in the distillation system. Due to the lack of mass and heat transfer correlations or experimental data to develop those correlations for the horizontal distillation system, mass (Bravo et al. 1985) and heat (Taylor, R., Krishna, 1993) transfer correlations that have been developed for distillation systems have been used to calculate the transport coefficients. The applied correlations consider distillation systems with packing material, and are used to calculate the transfer rate equations between the vapor and liquid phases in the rectifying and stripping sections. The transport coefficients investigated here are mass transfer coefficients for the vapor and liquid phase $\left(k^{L}, k^{\vee}\right)$, heat transfer coefficient $(h)$ and interfacial area $(\alpha)$. Figures 2-3 show the effect of the above-mentioned variables on reflux and boil-up ratio (which are related to energy requirements) in the stripping and rectifying section respectively, while the light product flowrate, recovery and purity have been kept constant. The x-axis of the Figures 2 and 3 , show the values of the factor $f$, which is used as a factor to multiply the initially calculated transport coefficients $k_{0}^{V}, k_{0}^{L}, h_{0}$, and $\alpha_{0}$ and manipulate their value. The factor $f$ can in principle take any non-zero positive value, if $f$ is less than 1 the transport coefficient is less than the initially calculated value $\left(k_{0}^{v}, k_{0}^{L}, h_{0}\right.$, and $\left.\alpha_{0}\right)$, while values of $f$ greater than 1 mean higher values compared to the initial ones. In this specific case, the initial transport coefficient values have been calculated considering packed material, therefore for a tray-less and without packing material system, values of the factor $f$ between zero and $1(0<f \leq 1)$ would make sense. The results (illustrated in Figures 2 and 3 ) show that only big deviations from the calculated initial $\left(k_{0}^{V}, k_{0}^{L}, h_{0}\right.$, and $\left.\alpha_{0}\right)$ values may affect the system performance in terms of energy requirements (represented here through boil-up and reflux ratio). More specifically, the analysis of the heat transfer coefficient in the stripper and rectifier shows that its effect is negligible for changes up to $95 \%$ while higher changes might slightly increase the energy required. Similar observations are made for the interfacial area and the liquid mass transfer while big changes in vapor mass transfer coefficient especially in the stripping section might lead to an increased energy consumption. 

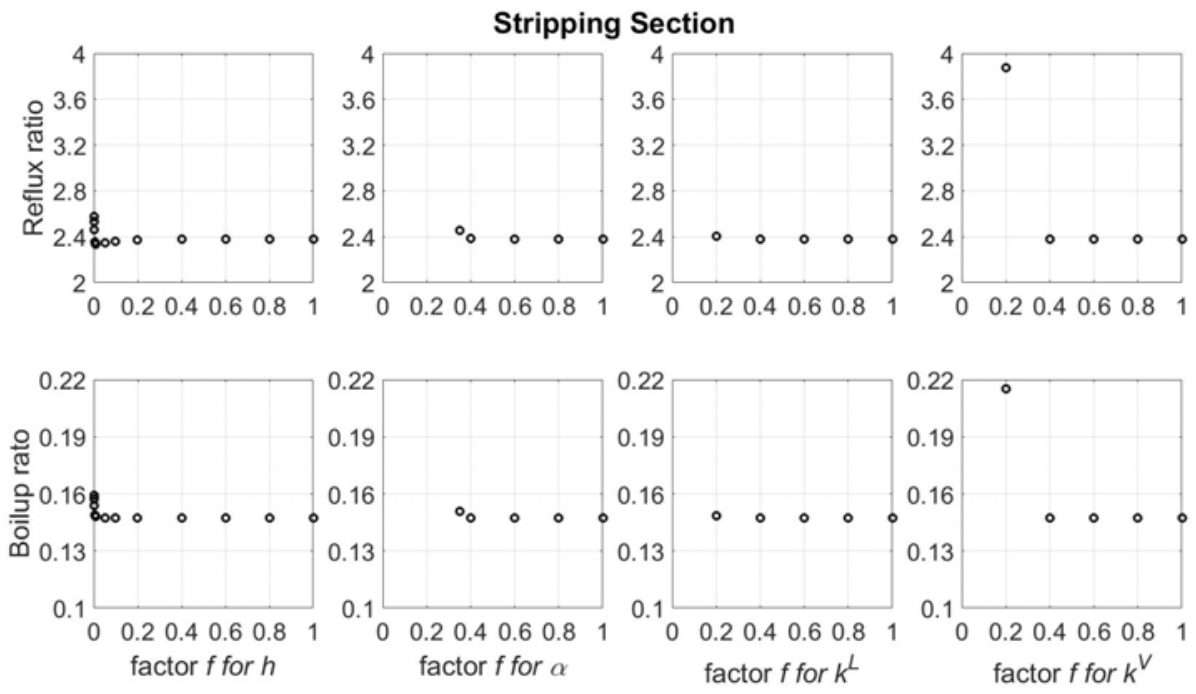

Figure 2: Transport coefficients sensitivity analysis for the stripping section. $k^{\vee}, k^{\perp}$ are mass transfer coefficients for the vapour and liquid phase respectively, $h$ is the heat transfer coefficient and $\alpha$ is the interfacial area.
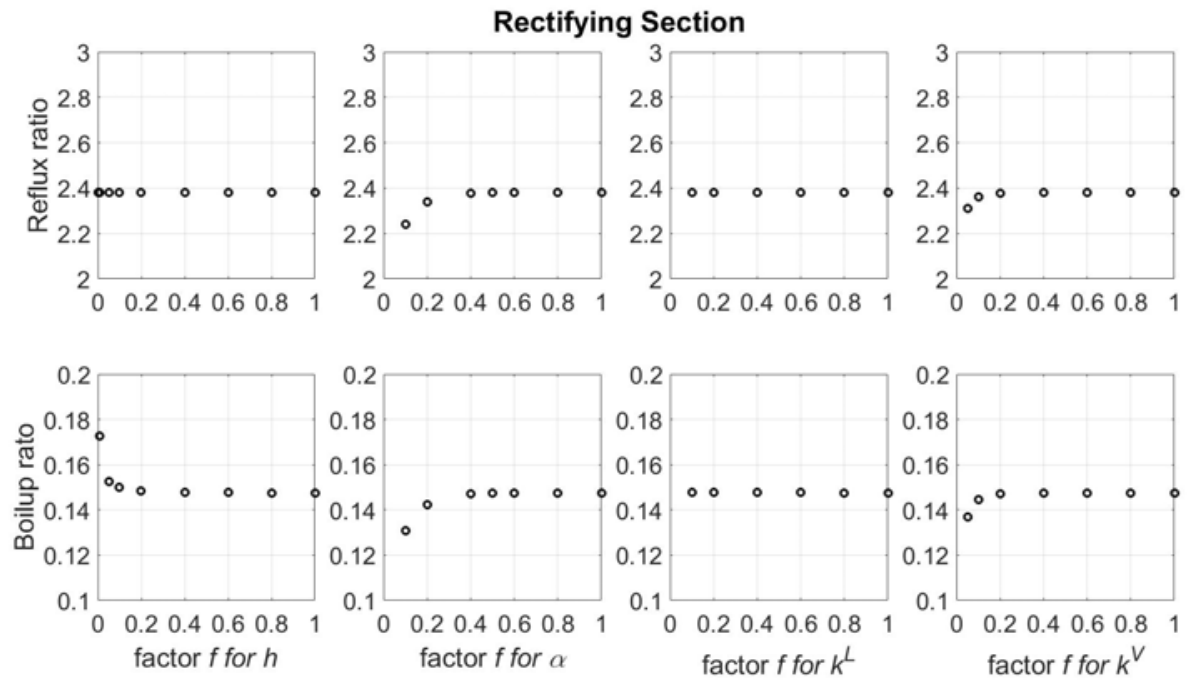

Figure 3: Transport coefficients sensitivity analysis for the rectifying section. $k^{v}$, $k^{L}$ are mass transfer coefficients for the vapour and liquid phase respectively, $h$ is the heat transfer coefficient and $\alpha$ is the interfacial area.

\section{Model application-Simulation studies}

\subsection{Recovery ratio and product purity}

The specific separation system is intended for separation of mixtures containing light alcohols, in small quantity, from heavier substances (e.g. water) in presence of valuable, temperature sensitive components such as enzymes. Therefore, it is valuable to investigate the dependence of the light alcohols recovery in the stripper and the light alcohols purity in the rectifier. For the ethanol-water mixture, Figure 4 shows the effect of changes in the specifications (recovery and purity) to operational parameters (reflux and boil-up ratio) as well as to performance criteria such as the energy requirements. Figure 4a shows that the boil-up rate remains almost constant for different product purities in the rectifier, but higher values of boil-up ratios are required when higher recovery ratios in the stripper are required. Similar observations are shown in Figure 4c and 4d for the energy requirements. Figure $4 b$ shows that the reflux ratio decreases when the recovery ratio is decreased. Moreover, it also shows that the reflux is decreased when the specified ethanol purity is decreased due to lower internal flows. 

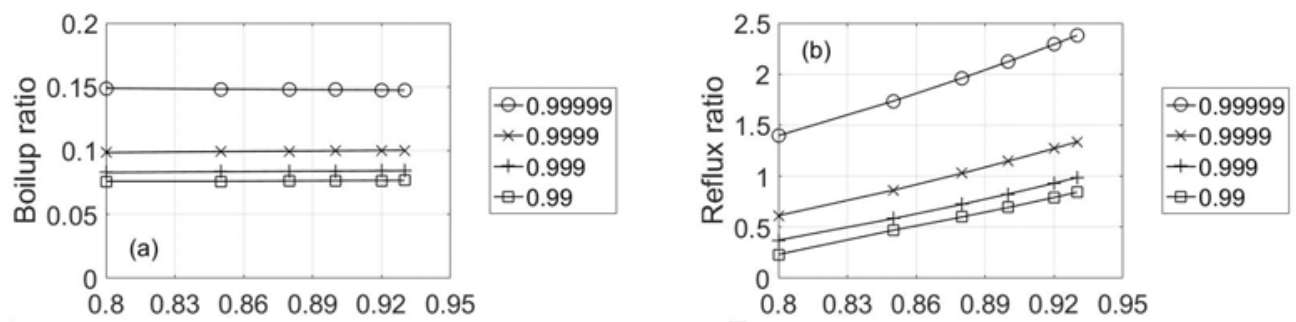

(a)

$\begin{array}{llllll}0.8 & 0.83 & 0.86 & 0.89 & 0.92 & 0.95\end{array}$

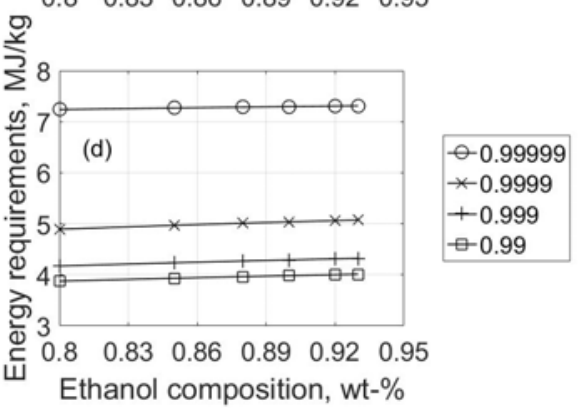

Figure 4: Dependence of operational variables and performance criteria with respect to ethanol product purities in the rectifying section (wt.-\%) for different recovery ratios of ethanol in the stripping section. (a) Boilup ratio, (b) reflux ratio, (c) energy requirements in $\mathrm{MJ} / \mathrm{h}$ and (d) energy requirements in $\mathrm{MJ} / \mathrm{kg}$ of product.

Figure 5 shows the dependence of the reflux ratio and energy requirements with respect to the ethanol recovery in the stripper for different product purities in the rectifier. Figures $5 a$ and $5 b$ show that energy requirements are reduced substantially when the recovery ratio in the stripper is lower, however, the energy requirements for different product purities in the rectifier (Figure 5b) do not vary substantially.
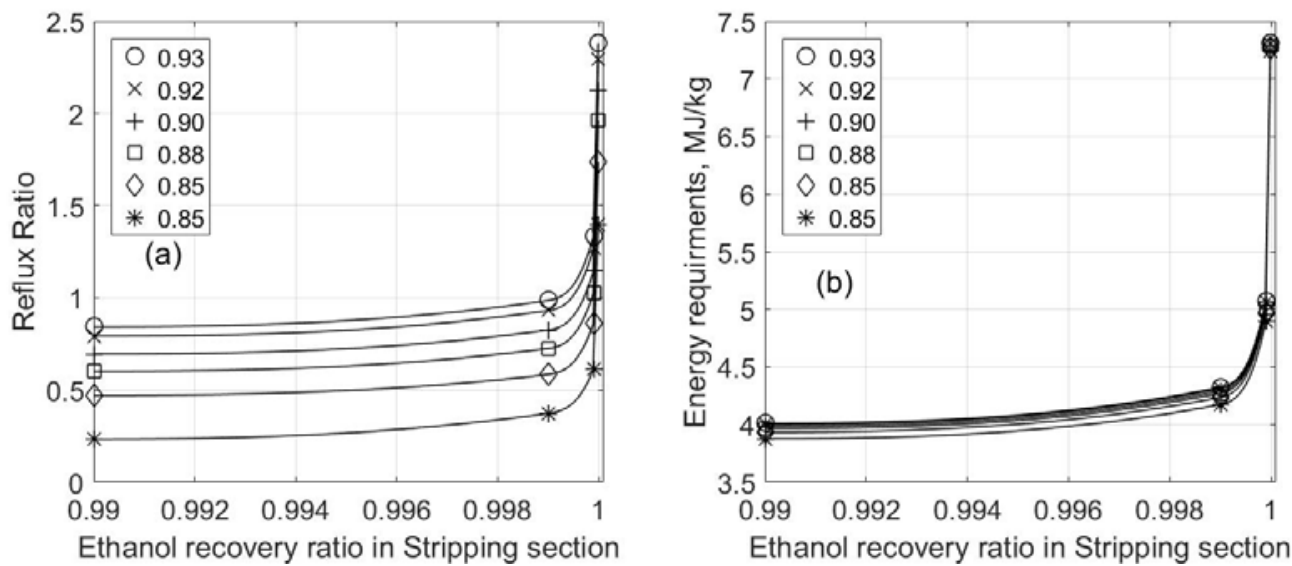

Figure 5: Dependence of operational variables and performance criteria with respect to the recovery ratio of ethanol in the stripper for different ethanol product purities in the rectifier. (a) Reflux ratio, (b) energy requirements in $\mathrm{MJ} / \mathrm{kg}$ of product.

\subsection{Heat transfer area}

An important design parameter is the heat transfer area between the rectifier and the stripper. The calculation of the heat transfer area has been explained briefly in Section 3.1. The variable $n$ has been added in the model (Eq. 1) to show the effect of the heat transfer area on the performance of the system in terms of operational variables and energy requirements. Figure 6 illustrates such analysis for $n=1$, 2, and 3. It is clearly seen that for a specified high ethanol recovery ratio in the rectifier (0.99999) the energy requirements for higher heat transfer areas have been decreased. It is also seen that the operational variables (Figure 6a and $6 \mathrm{~b}$ ) have been significantly decreased, which means that further increase of the heat transfer area might be unrealistic from an operational point of view as boil-up and reflux ratio will have very low values to achieve the separation objectives. 

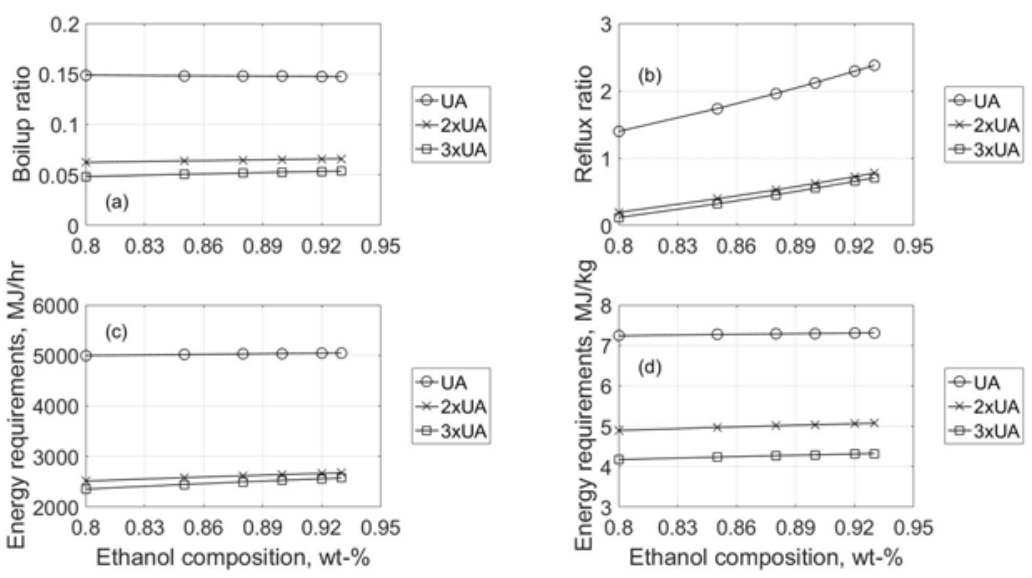

Figure 6: Dependence of operational variables and performance with ethanol purities (wt.-\%) in the rectifier and heat transfer area. (a) Boilup and (b) Reflux ratio, (c) Energy requirements in MJ/h and (d) in MJ/kg of product.

\section{Conclusions}

In this paper, an adiabatic horizontal distillation system, intended for small-scale separations of chemicals from $2 G$ bio-refineries and a steady state model that provides a meaningful description, in absence of experimental data, has been described. The model has been applied in sensitivity analysis of the transport coefficients and it has been shown that the effect of the heat transfer coefficient, the interfacial area and the liquid mass transfer and changes below $60 \%$ of the initial vapor mass transfer coefficient value are negligible. The model has also been applied in simulation studies evaluating the operational variables and performance criteria for different process specifications. Previously, potential improvements in terms of energy requirements reduction over conventional separation schemes in mixtures containing small amounts of light alcohols have been shown as proof of concept studies (Papadakis et al. 2018). Finally, an economic model taking into account the capital cost of the system including a compressor, a condenser and a reboiler as well as the operational costs is required. The economic model in combination with the steady state model will be used in process optimization with the objective to minimize the total costs subject to heat transfer area and compression ratio. Finally, complementary work has been planned to investigate the dynamics and the operability of the process.

\section{Acknowledgments}

The authors would like to acknowledge the financial support of the SYNFERON (journal no. 4106-00035B by Innovation Fund Denmark) project and Morten J.G. Larsen for designing Figure 1.

\section{References}

Bravo J.L., Rocha J.A., Fair J.R., 1985, Mass transfer in gauze packings, Hydrocarbon Processes, 64, 91-95. Jang D. J., \& Kim, Y. H., 2015, A new horizontal distillation for energy saving with a diabatic rectangular column. Korean Journal of Chemical Engineering, 32, 2181-2186.

Kim B. C., Chun H. H., Kim Y. H., 2013, Energy-efficient diabatic distillation using a horizontal distillation column. Industrial and Engineering Chemical Research, 52, 14927-14935.

Kiss A. A., 2014, Distillation technology - still young and full of breakthrough opportunities, Journal of Chemical Technology and Biotechnology, 89, 479-498.

Maity S. K., 2015, Opportunities, recent trends and challenges of integrated biorefinery: Part I, Renewable and Sustainable Energy Reports, 43, 1427-1445.

Papadakis E., Mansouri S. S., Huusom K. J., Abildskov J., Analysis and evaluation of a heat integrated horizontal distillation system, Proceedings of the $13^{\text {th }}$ International Symposium on Process Systems Engineering - PSE 2018, July 1-5, 2018, San Diego, California (accepted).

Ramirez-Gonzalez E. A., Martinez C., Alvarez J., 1992, Modeling Zero-Gravity Distillation, Industrial and Engineering Chemical Research, 31, 901-908.

Seok D. R., Hwang S. T., 1985, Zero-Gravity Distillation Utilizing the Heat Pipe Principle (Micro-distillation), AIChE Journal, 31, 2059-2065.

Taylor R., Krishna R., 1993, Multicomponent Mass Transfer, New York. 